\title{
Distúrbios osteomusculares e ações para reduzir a ocorrência em trabalhadores de enfermagem
}

\author{
Musculoskeletal disorders and actions to reduce the occurrence in nursing staff \\ Trastornos musculoesqueléticos y acciones para reducir la ocurrencia en trabajadores de enfermería
}

Silmar Maria da Silva'; Natália Teixeira Braga"; Rosimeire Ângela de Queiroz Soares ${ }^{\prime \prime \prime}$; Patricia Campos Pavan Baptista ${ }^{\prime V}$

\begin{abstract}
RESUMO
Objetivo: identificar a presença de distúrbios osteomusculares relacionados ao trabalho nos trabalhadores de enfermagem de uma unidade de clínica médica e construir juntos aos trabalhadores propostas para reduzir a ocorrência dos distúrbios osteomusculares no ambiente de trabalho. Método: estudo transversal com 31 trabalhadores de enfermagem de uma clínica médica, que responderam uma ficha de dados sóciodemográfico e profissional e do Questionário Nórdico de Sintomas Osteomusculares. Resultados: os trabalhadores exercem suas atividades laborais com dores osteomusculares, sendo as regiões corpóreas mais prevalentes a lombar e a porção superior da coluna e ombros. Apesar disso, nem todos se afastam do trabalho ou procuram assistência terapêutica. As ações de redução dos distúrbios osteomusculares levantadas foram categorizadas em três dimensões: indivíduo, equipe e instituição. Conclusão: os trabalhadores apresentam distúrbios osteomusculares, principalmente, nas costas. As ações de redução de sua ocorrência perpassam pelo comportamento individual às mudanças estruturais e provisão de equipamentos de trabalho.
\end{abstract}

Descritores: Transtornos Traumáticos Cumulativos; Prevenção de Doenças; Enfermagem; Saúde do Trabalhador.

\section{ABSTRACT}

Objective: to identify the presence of work-related musculoskeletal disorders in nursing staff at an internal medicine unit and, jointly with the staff, to build proposals to reduce the occurrence of musculoskeletal disorders in the workplace. Method: in this cross-sectional study, 31 nursing staff of a medical clinic answered a socio-demographic and professional data sheet and the Nordic Musculoskeletal Questionnaire. Results: staff performed their work activities with musculoskeletal pain, the most prevalent body regions being: lower back and upper spine and shoulders. Nonetheless, not everyone would take time off work or seek therapeutic care. The actions suggested to reduce musculoskeletal disorders were categorized into three dimensions: individual, team and institutional. Conclusion: staff had musculoskeletal disorders, mainly in the back. Actions proposed to reduce pain ranged from individual behavior to structural changes and provision of work equipment.

Descriptors: Cumulative Trauma Disorders; Disease Prevention; Nursing; Occupational Health.

\section{RESUMEN}

Objetivo: identificar la presencia de trastornos musculoesqueléticos relacionados con el trabajo en el personal de enfermería en una unidad de medicina interna y, conjuntamente con el personal, elaborar propuestas para reducir la aparición de trastornos musculoesqueléticos en el lugar de trabajo. Método: en este estudio transversal, 31 miembros del personal de enfermería de una clínica médica respondieron una hoja de datos sociodemográficos y profesionales y el Cuestionario musculoesquelético nórdico. Resultados: el personal realizó sus actividades laborales con dolor musculoesquelético, siendo las regiones corporales más frecuentes: la parte baja de la espalda y la parte superior de la columna y los hombros. Sin embargo, no todos tomarían tiempo libre del trabajo o buscarían atención terapéutica. Las acciones sugeridas para reducir los trastornos musculoesqueléticos se clasificaron en tres dimensiones: individual, de equipo e institucional. Conclusión: el personal tenía trastornos musculoesqueléticos, principalmente en la espalda. Las acciones propuestas para reducir el dolor iban desde el comportamiento individual hasta los cambios estructurales y la provisión de equipos de trabajo.

Descriptores: Trastornos de Traumas Acumulados; Prevención de Enfermedades; Enfermería; Salud Laboral.

\section{INTRODUÇÃO}

Os trabalhadores de enfermagem frequentemente se encontram em posturas inadequadas durante a jornada de trabalho por permanecerem em pé por um longo período de tempo ou por realizarem atividades que exigem a curvatura corporal. Por vezes, realizam atividades que requer esforço físico, como mudança de decúbito ou auxílio ao paciente para deixar o leito. Estas características ocupacionais levam a uma elevada prevalência de Distúrbios Osteomusculares Relacionados ao Trabalho (DORT) entre estes profissionais ${ }^{1}$.

'Enfermeira. Doutora. Professora Adjunta. Universidade Federal de Minas Gerais. Belo Horizonte, Brasil. silmarmaria@uol.com.br. ORCID: https://orcid.org/0000-0002$8322-3917$

"Enfermeira. Residência em Enfermagem Clínico-Cirúrgica. Hospital Sírio-Libanês. São Paulo, Brasil. nataliatb62@gmail.com. ORCID: https://orcid.org/0000-0002-8948-3330 I'Enfermeira. Doutora. Professora Instrutora. Faculdade de Ciências Médicas da Santa Casa de São Paulo. Brasil. roseenfh@gmail.com. ORCID: https://orcid.org/00000002-3752-6634

IVEnfermeira. Doutora. Professora Titular. Universidade de São Paulo, São Paulo, Brasil. pavanpati@usp.br. ORCID: https://orcid.org/0000-0003-1433-6456

vo presente estudo recebeu apoio da Pró-Reitoria de Pesquisa (PRPq) da Universidade Federal de Minas Gerais. 
Os DORT estão entre os agravos que mais afetam os trabalhadores brasileiros. Trata-se de uma síndrome clínica, de origem multifatorial complexa, que envolve aspectos individuais e da organização do trabalho, de aparecimento e evolução de caráter insidioso, geralmente progressivo, permanente ou não, com repercussão fisiológica oriunda de processos de desgaste sofridos pelo sistema osteomuscular, sem que haja tempo hábil para sua devida recuperação ${ }^{2}$.

Os DORT apresentam como principal característica a dor crônica, espontânea ou por movimento. Também podem estar presentes sensação de dormência, formigamento, alteração na sensibilidade, agulhadas, choques, exaustão muscular, entre outros ${ }^{2}$.

O comprometimento osteomuscular ocorre prioritariamente durante o exercício profissional, que exige disponibilidade física para caminhadas intermitentes, suporte de cargas mecânicas, como manipulação de paciente, longos períodos em posição ortostática, necessidade de atenção constante. Somados a isto, pode-se encontrar postos de trabalhos e enfermarias não adequados ergonomicamente às exigências do exercício profissional ${ }^{3}$, como alturas de pias e de bancada de trabalho inadequadas, necessidade frequente de abaixar-se ou ficar na ponta do pé para alcançar materiais e insumos, cadeiras e monitores de computador sem a possibilidade de ajustes, ou cadeira sem encosto, leitos muito próximos um dos outros, banheiros pequenos, corredores longos, e rodízios de carrinhos de banho, cadeira higiênica e macas sem regular manutenção preventiva.

Em consequência, os DORT desencadeiam um processo de desgaste físico e mental, com repercussão na prática profissional e comprometimento do rendimento durante o período de trabalho. Pode ainda reverberar no âmbito social da vida deste indivíduo, tendo em vista que as dores/desconfortos podem associar-se às limitações funcionais ${ }^{4}$.

Os DORT são uma das principais causas de afastamento do trabalho, com afastamentos que ocorrem por períodos inferiores a 15 dias, de forma recorrente e pelos mesmos motivos, acumulando uma grande quantidade de dias perdidos de trabalho de até 325 dias/12 meses $^{5}$.

Frente a essas considerações, este estudo busca oferecer subsídios para que as instituições hospitalares possam compreender que o adoecimento no ambiente de trabalho impacta na vida do trabalhador e que as propostas de intervenção, na tentativa de minimizar os agravos na saúde dos trabalhadores, devem sem pautadas a partir do olhar de quem experencia as condições laborais inadequadas. Neste sentido, este estudo teve como objetivo identificar a presença de distúrbios osteomusculares relacionados ao trabalho nos trabalhadores de enfermagem de uma unidade de clínica médica e construir juntos aos trabalhadores propostas para reduzir a ocorrência dos distúrbios osteomusculares no ambiente de trabalho.

\section{MÉTOdO}

Trata-se de um estudo exploratório, descritivo, de corte transversal, com abordagem quantitativa, realizado em uma unidade de clínica médica, com 28 leitos, de um hospital universitário no estado de Minas Gerais. Destes, três enfermarias, com dois leitos cada, eram utilizadas para isolamento de contato, social ou como leito psiquiátrico e os demais leitos eram destinados aos pacientes provenientes do Pronto Atendimento, em acompanhamento pelas clínicas médica, de infectologia, da neurologia, da oncologia ou da cardiologia. Para definição da divisão trabalho, nomeada por escala de trabalho diária, os pacientes eram classificados por grau de complexidade, segundo a mobilidade, o tempo necessário para prestar os cuidados, quantidade e complexidade das medicações que seriam administradas. Embora a unidade fosse fisicamente pequena, ainda era considerado a distância entre as enfermarias para a divisão do trabalho.

Para participar do estudo foi adotado como critério de inclusão trabalhar na instituição no mínimo há seis meses. Foram excluídos os que estavam de folga, férias ou afastado do trabalho por licença médica ou maternidade no período da coleta de dados. Assim, dos 34 trabalhadores que compunham a equipe de enfermagem da unidade, foram excluídos dois técnicos de enfermagem em férias e uma técnica de enfermagem em licença maternidade. Todos os trabalhadores elegíveis foram convidados e aceitaram participar do estudo. A amostra foi composta por sete enfermeiros, 22 técnicos de enfermagem e dois auxiliares de enfermagem, totalizando 31 participantes, a partir de uma amostra homogênea, intencional, composta pela totalidade dos profissionais da enfermagem.

A coleta ocorreu entre os meses de fevereiro e março de 2019, no local de trabalho. Foram entregues, em envelope pardo com decodificação alfanumérica, a ficha de dados sóciodemográfico e profissional com uma questão aberta: Para reduzir a ocorrência dos distúrbios osteomusculares, quais medidas deveriam ser adotadas no ambiente de trabalho (por você, colegas e/ou chefia)?, e o Questionário Nórdico de Sintomas Osteomusculares (QNSO). Foi acordado que o envelope seria devolvido aos pesquisadores no próximo plantão.

O QNSO foi desenvolvido na Finlândia ${ }^{6}$, validado ${ }^{7}$ e adaptado para a cultura brasileira ${ }^{8}$, e tem como objetivo avaliar a presença de sintomas osteomusculares em profissionais, como dor, formigamento e dormência. Tem sido reconhecido internacionalmente para avaliar a presença de sintomas de $\mathrm{DORT}^{9}$. Contém uma figura humana vista pela região posterior, dividida em nove regiões: cervical, ombros, torácica, cotovelos, punhos/mãos, quadril/coxas, joelhos e 
tornozelos/pés. As questões estão relacionadas com cada área anatômica e verificam se os respondentes tiveram dor, formigamento/dormência nos últimos 12 meses e nos últimos sete dias. Procuram também investigar se estas pessoas precisaram faltar ao serviço ou procurar auxílio médico nos últimos 12 meses devido aos mesmos sintomas ${ }^{8}$.

Os dados foram inseridos na planilha do programa de computador Microsoft Office Excel ${ }^{\circledR}$ 2016, em forma de banco de dados eletrônico, com dupla digitação independente. Após a correção de erros e inconsistências, os dados foram exportados para o Software Statistical Package for the Social Sciences for Windows (SPSS ${ }^{\circledR}$ ) versão 19.0, estabelecendo-se as análises descritivas, a partir de médias, frequências absolutas e relativas. E os dados subtraídos da questão aberta foram analisados, categorizados e tratados quantitativamente.

O estudo seguiu as recomendações da Resolução no 466/12 do Conselho Nacional de Saúde, sendo seu projeto aprovado pelo Comitê de Ética em Pesquisa sob parecer no 2.673.822. Os participantes foram esclarecidos sobre a pesquisa e assinaram o Termo de Consentimento Livre e Esclarecido.

\section{RESULTADOS}

Participaram do estudo 31 trabalhadores, com taxa de resposta de 91,1\%, preponderando o sexo feminino (64,5\%), com uma de média de $40 \pm 9,85$ anos de idade, predominantemente, entre 25 a 44 anos de idade (71,0\%). A maioria formada por técnicos de enfermagem (71,0\%), atuando no turno noturno (61,3\%), com vínculo único de trabalho $(87,1 \%)$, com uma média de $36,0 \pm 8,6$ horas de trabalho semanal e um tempo médio de exercício da profissão de $16,0 \pm 9,26$ anos, sendo que 14 (45,2\%) exercem a profissão entre 11 a 20 anos. E de acordo com o Índice de Massa Corpórea (IMC), a maior parte apresentava sobrepeso ou obesidade $(61,3 \%)$.

Em relação ao QNSO, a maioria dos participantes referiu apresentar sintomas osteomusculares, em uma ou mais partes do corpo, sendo que $27(87,0 \%)$ nos últimos 12 meses e 17 (54,8\%) nos últimos sete dias.

Nos últimos 12 meses, houve predomínio dos sintomas osteomusculares na região inferior das costas (58,1\%), superior das costas e ombros (54,8\%), seguidas pelas regiões pescoço, punhos/mãos e joelhos (45,2\%). E nos últimos sete dias, as regiões mais relatadas foram: inferior das costas $(29,0 \%)$, superior das costas $(25,8 \%)$ e ombros e tornozelos/pés $(19,4 \%)$, como observado na Figura 1.

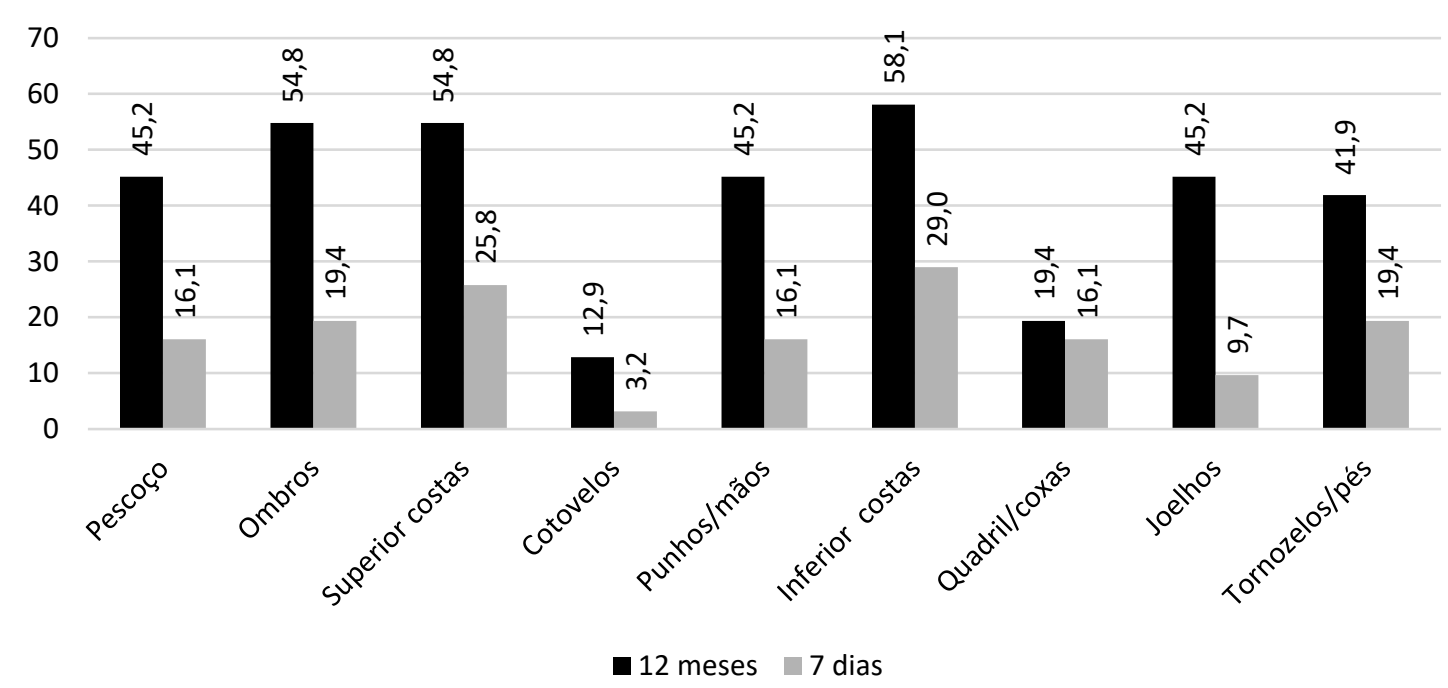

Figura 1: Frequência (\%) de sintomas musculoesqueléticos relatados pelos trabalhadores de enfermagem, nos últimos 12 meses e nos últimos sete dias, segundo as diferentes regiões corpóreas (n=31). Belo Horizonte, Brasil, 2019.

Apesar dos sintomas osteomusculares nas costas e ombros serem os mais prevalentes, os sintomas que mais levaram ao impedimento de realizar atividades foram os que acometeram quadril/coxas (50,0\%) e pescoço e punhos/mãos (42,8\%). Em relação a busca por um profissional de saúde, houve maior proporção entre os que apresentaram sintomas em punhos/mãos (50,0\%), como apresentado na Figura 2. 


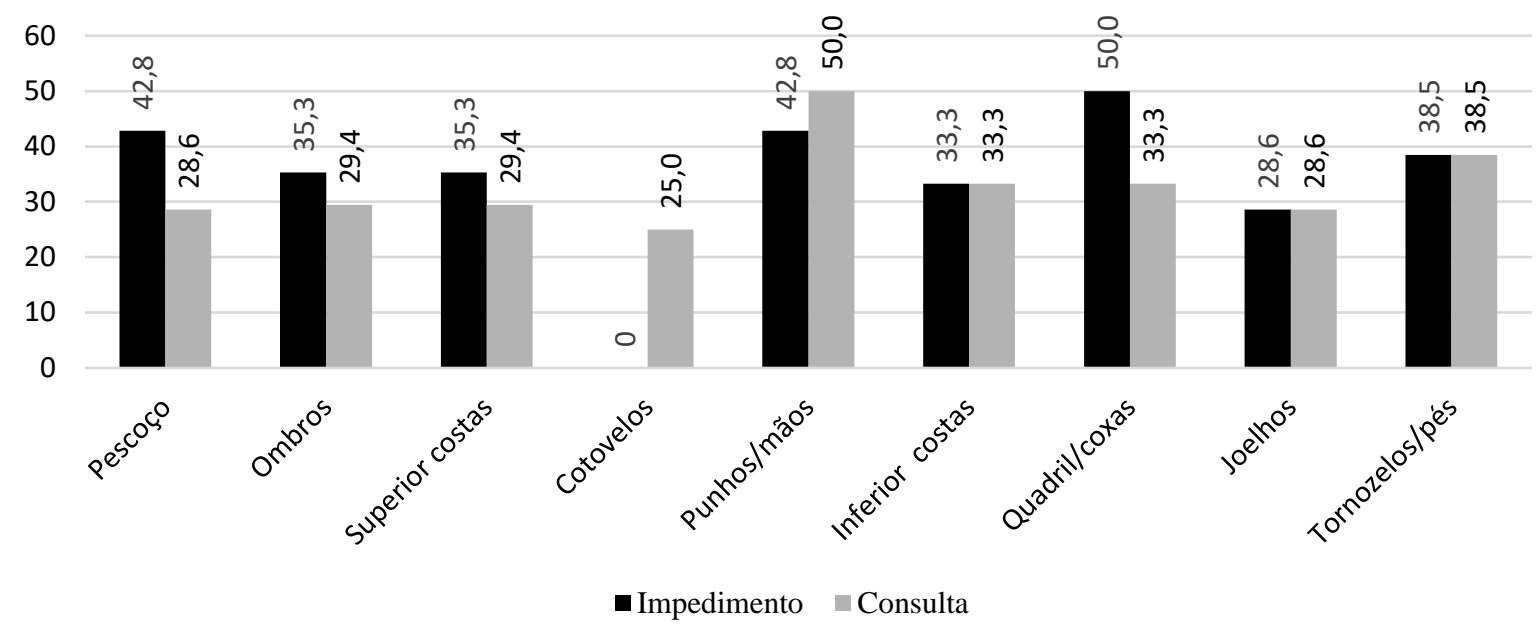

Figura 2: Frequência (\%) de impedimento de realizar atividades e de consulta ao profissional de saúde entre os trabalhadores de

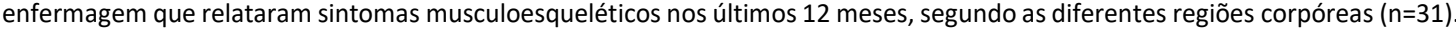
Belo Horizonte, Brasil, 2019.

As propostas levantadas entre os trabalhadores de enfermagem, para reduzir a ocorrência dos DORT, foram analisadas e categorizadas em três dimensões: indivíduo, equipe e instituição, como apresentado na Figura 3.

\begin{tabular}{|c|c|c|c|c|}
\hline \multicolumn{5}{|c|}{ Indivíduo } \\
\hline \multicolumn{5}{|c|}{$\begin{array}{l}\text { - Uso adequado das camas; } \\
\text { - Postura correta no atendimento ao paciente evitando curvatura na coluna; } \\
\text { - Manter atenção redobrada em todas as atividades a serem realizadas; } \\
\text { - Realizar as funções com maior calma e tempo; } \\
\text { - Participar dos treinamentos oferecidos pela instituição. }\end{array}$} \\
\hline \multicolumn{5}{|c|}{ Equipe } \\
\hline \multicolumn{5}{|c|}{$\begin{array}{l}\text { - Manter o ambiente limpo e organizado; } \\
\text { - Boa convivência, comunicação clara e eficiente entre os membros da equipe; } \\
\text { - Cooperação entre colegas para tarefas que exijam esforço em equipe ou dupla. }\end{array}$} \\
\hline \multicolumn{5}{|c|}{ Instituição } \\
\hline & Mobiliário/ Estrutura & Recursos Humanos & & Capacitação \\
\hline & $\begin{array}{l}\text { Condições adequadas dos } \\
\text { equipamentos; } \\
\text { Mobiliários adequados e em } \\
\text { número suficiente; } \\
\text { Espaço físico adequado para } \\
\text { desenvolvimento das } \\
\text { atividades; } \\
\text { lluminação adequada; } \\
\text { Manutenção periódica dos } \\
\text { equipamentos com rodas; } \\
\text { Aquisição de equipamentos } \\
\text { para reduzir a força física na } \\
\text { movimentação do paciente. }\end{array}$ & $\begin{array}{l}\text { - Manter o quadro de funcionários } \\
\text { com o quantitativo adequado para a } \\
\text { demanda do setor; } \\
\text { - Diminuir o número de pacientes } \\
\text { atendidos por profissional; }\end{array}$ & & $\begin{array}{l}\text { Treinamentos sobre } \\
\text { ergonomia durante o banho } \\
\text { no leito, na mudança de } \\
\text { decúbito, como pegar peso, } \\
\text { etc. } \\
\text { Palestras e cursos sobre } \\
\text { segurança e bem-estar do } \\
\text { funcionário; } \\
\text { Educação permanente. }\end{array}$ \\
\hline
\end{tabular}

Figura 3: Ações para prevenção dos sintomas musculoesqueléticos, segundo os trabalhadores de enfermagem da unidade de clínica médica. Belo Horizonte, Brasil, 2019. 


\section{DISCUSSÃO}

Ao investigar a presença de DORT nos trabalhadores de enfermagem de uma unidade de clínica médica, foi identificado uma amostra majoritariamente feminina (64,5\%) e jovem, com média de $40 \pm 9,85$ anos de idade. A ocorrência de DORT tem sido registrada com maior frequência em trabalhadores do sexo feminino, como apontado em alguns estudos ${ }^{10-11}$, contudo, o mecanismo envolvido ainda não está elucidado.

O tempo médio de exercício da profissão foi de 16,0 \pm 9,26 anos, bem próximo do tempo médio de 13 anos de um estudo português ${ }^{12}$. As tarefas da enfermagem e a frequência em que são realizadas estão entre os fatores determinantes para o surgimento dos DORT, mesmo em uma população mais jovem.

Os técnicos de enfermagem são os mais susceptíveis à dor osteomuscular, com 2,794 mais chances em relação aos outros profissionais da saúde, relatando mais dores, fadiga e tensão. Já os enfermeiros referem mais tensão muscular ${ }^{13}$.

O turno noturno tem relação positiva com ganho de peso entre os trabalhadores de enfermagem, por razões de alterações hormonais e sociais ${ }^{14}$, como evidenciado em estudo com 671 enfermeiros que correlacionou a ocorrência de dor lombar aguda ao turno noturno, turnos prolongados e obesidade ${ }^{15}$. Além do mais, estes fatores contribuem para a elevação do IMC, com impacto na prevalência da dor lombar ${ }^{16}$.

Os DORT possuem alta ocorrência entre os profissionais de enfermagem. Foi evidenciado que 27(87,0\%) trabalhadores de enfermagem apresentaram sintomas osteomusculares nos últimos 12 meses e 17 (54,8\%) nos últimos sete dias. Resultados similares foram encontrados em outros estudos que averiguarem sintomas osteomusculares nos últimos 12 meses $\left(88,0 \%{ }^{16}, 82,1 \%{ }^{17}\right.$ e $\left.74,7 \%{ }^{18}\right)$, e nos últimos sete dias $\left(58,0 \%{ }^{16}\right)$.

Houve predomínio dos sintomas osteomusculares nas costas tanto nos últimos 12 meses quanto nos últimos sete dias, particularmente na região inferior das costas, a região lombar. Em uma coorte com 4.977 profissionais de saúde foi identificado que o esforço físico, percebido extenuante durante o trabalho em saúde, aumentou o risco de dor crônica na lombar (OR 3,16, IC 95\% 1,79-5,57) e no joelho (OR 1,87, IC95\% 1,19-2,94) $)^{19}$. Além disso, a lombar ${ }^{19-20}$ tem sido a região corpórea mais referida entre os trabalhadores com desconforto osteomuscular, seguida pela cervical, torácica $^{20}$, pescoço e joelhos ${ }^{21}$.

A dor lombar é resultante do aumento da pressão na coluna vertebral e está relacionada à natureza do trabalho de enfermagem e às técnicas de elevação inadequadas do paciente ${ }^{22}$, ao levantamento de cargas excessivas, às constantes flexões na coluna e às posturas inadequadas durante os procedimentos ${ }^{11}$.

Alguns estudos têm demonstrado que, além dos impactos na vida profissional e social do trabalhador de enfermagem, os DORT, particularmente a dor lombar crônica ${ }^{23}$, apresentam relação com o presenteísmo ${ }^{24}$, condição na qual os trabalhadores comparecem ao trabalho doentes, gerando queda na produtividade, com consequentes riscos à sua integridade e à segurança do paciente ${ }^{25}$. O absenteísmo (ausência e/ou atrasos no trabalho) também é citado na literatura como uma das consequências da ocorrência de DORT nos profissionais ${ }^{16}$.

Logo, os trabalhadores de enfermagem têm permanecido no ambiente de trabalho, prestando assistência, ainda que com dor. E a dor, particularmente na região lombar não tem sido impeditiva para realizar as atividades e nem motivo para buscar atendimento de um profissional, ao menos entre os participantes deste estudo. Por outro lado, esta situação pode comprometer a prática profissional, como constatado em estudo em que $81,8 \%$ dos trabalhadores de enfermagem reconheceram que trabalhar mesmo sentindo dor é um grande problema para o processo de trabalho ${ }^{26}$.

Diferentes motivos, como a invisibilidade dos DORT, o medo do desemprego, os estigmas, ausência de suporte social no trabalho ${ }^{27}$, ou mesmo a naturalização da dor, a automedicação, a auto cobrança, o compromisso com a equipe - em não desfalcá-la, podem levar o trabalhador de enfermagem a permanecer no campo de trabalho, o que contribui para o agravamento e cronificação da sua condição clínica, com repercussões importantes, como a piora da dor, a limitação dos movimentos à incapacidade física, e a necessidade de afastar-se do trabalho de maneira permanente.

Assim, na tentativa de minimizar os agravos à saúde dos trabalhadores e reduzir a ocorrência dos DORT, foi proposto um conjunto de ações no ambiente de trabalho destinado ao trabalhador individualmente, à equipe e à instituição, evidenciando a corresponsabilidade dos trabalhadores e da instituição.

$\mathrm{Na}$ dimensão individual, foram propostas ações que perpassam pelo uso adequado do mobiliário, postura adequada, atenção e participação nos treinamentos. As camas da unidade de clínica médica eram todas elétricas, contudo, nem todos os profissionais utilizavam os seus recursos. Assim, se propõe o uso adequado da cama, ajustando a altura mais confortável ao trabalhador na assistência à beira leito, evitando a curvatura da coluna. Para tanto, cabe ao profissional maior planejamento do tempo para executar as atividades com maior atenção e calma, e comprometimento em participar dos treinamentos oferecidos pela instituição. 
Na dimensão da equipe, as ações movem-se para a boa relação entre os trabalhadores, a partir do cuidado do ambiente comum, mantendo-o limpo e organizado, da boa comunicação na equipe e da cooperação nas atividades que exigem esforço físico. As relações não saudáveis entre os membros da equipe de enfermagem podem gerar conflitos e relações interpessoais difíceis entre a equipe, contribuindo para o adoecimento do trabalhador. Deste modo, deve-se subsidiar ações que minimizem o processo de desgaste do trabalhador ${ }^{28}$.

Quanto às ações que competem a dimensão instituição, estas foram organizadas em três frentes: mobiliário/estrutura, recursos humanos e capacitação.

Enquanto que na dimensão individual cabia ao trabalhador fazer uso adequado dos recursos dos mobiliários, é de responsabilidade da instituição fornecer mobiliários e equipamentos adequados e em número suficiente, realizar a manutenção preventiva dos equipamentos e prover iluminação e espaço físico adequado para um trabalho seguro. Além disto, em razão do número de pacientes dependentes, propõe aquisição de equipamentos para reduzir a força física na movimentação do paciente.

Além do mais, cabe a instituição dar os recursos necessários para o cumprimento das atividades laborais de forma adequada e confortável, como mobiliário adequado, condizentes com as medidas antropométricas do profissional, com vistas a não acarretar problemas posturais e de movimentação ${ }^{29}$.

Todavia, apenas possuir os dispositivos de transferência não promove a segurança do trabalhador, pois é necessário criar um clima de conscientização na equipe de profissionais acerca das práticas seguras durante a transferência de pacientes. Também é preciso reduzir os empecilhos ao uso de tais dispositivos, como facilitar o acesso aos mesmos, disponibilizar locais adequados para seu armazenamento e espaço suficiente para seu uso ${ }^{30}$. Pois, ao contrário, haverá barreiras que pesarão na decisão de usar ou não os dispositivos de transferência.

Outras barreiras também podem estar presentes no ambiente de trabalho, como a dificuldade de acessar fisicamente os equipamentos e a falta de conhecimento e habilidade para o uso. Assim, é necessário que ocorra o envolvimento da equipe a favor do uso de tais dispositivos e o engajamento das instituições em melhorar o acesso e a capacitação dos profissionais ${ }^{31}$.

Para mais, podem ser aplicadas técnicas ergonômicas para reduzir o esforço físico exigido no manuseio dos pacientes, além de realizar a avaliação da carga de trabalho, das posturas e ergonômica do trabalho ${ }^{32}$.

Da mesma forma, a gestão dos serviços deve ser responsável pela organização e planejamento de estrutura física adequada, buscando evitar que os trabalhadores percorram longas distâncias nas instituições ${ }^{28}$.

Somado a isso, sugere o ajuste do número de trabalhadores de enfermagem de acordo com a demanda do setor e a redução do número de pacientes por profissional, com vistas a um número de trabalhadores suficientes para o desenvolvimento das atividades, sem a necessidade realizar esforço físico em excesso ${ }^{28}$.

Todavia, os trabalhadores de enfermagem, a fim a atender as demandas do serviço, submetem-se ao esforço físico, ao ritmo intenso e a repetitividade, sobrepondo, por vezes, seus limites físicos e mentais, e mantendo-se no ambiente de trabalho, mesmo na presença da dor osteomuscular ${ }^{27}$.

E, por fim, os participantes da pesquisa pontuam a importância da educação permanente, com vistas a formação sobre segurança e bem-estar do trabalhador, além de treinamentos sobre princípios da ergonomia na assistência ao paciente no banho no leito, na mudança de decúbito e no levantamento de peso.

Mesmo diante da magnitude da problemática dos sintomas osteomusculares em trabalhadores de enfermagem, evidentes neste estudo e na literatura nacional e internacional, existem tímidos programas de intervenção ergonômica, de cursos de treinamento do profissional para transferência de pacientes e de dispositivos mecânicos para transferência de pacientes ${ }^{11}$.

Em um estudo caso-controle randomizado, 67 enfermeiros do grupo caso participaram por três meses de atividades didáticas educativas, exercícios de fortalecimento da coluna e treinamento sobre técnicas seguras na elevação de pacientes. O escore médio de intensidade da dor lombar pré-intervenção na escala visual analógica diminuiu de 49,3 para o escore pós-intervenção de 7,5 (de 0 a 100). A correta execução das técnicas de elevação vertical no grupo experimental aumentou de $8,9 \%$ a $97,0 \%$. A técnica de elevação horizontal do paciente pré-intervenção aumentou de $10,4 \%$ a $100,0 \%$ de execução correta no grupo experimental ${ }^{22}$.

\section{CONCLUSÃO}

Os DORT são prevalentes entre os participantes da pesquisa, sendo que $87,0 \%$ referiram apresentar um ou mais sintomas, em alguma parte do corpo, nos últimos 12 meses, 54,8\% nos últimos sete dias. Em relação a localização, nos últimos 12 meses, preponderaram os sintomas osteomusculares na região inferior das costas (58,1\%), superior das costas 
e ombros (54,8\%), seguidas pelas regiões pescoço, punhos/mãos e joelhos (45,2\%). Nos últimos sete dias, as regiões mais relatadas foram: inferior das costas (29,0\%), superior das costas $(25,8 \%)$ e ombros e tornozelos/pés $(19,4 \%)$.

Quanto às propostas para reduzir os distúrbios osteomusculares no ambiente de trabalho sugeridas pelos trabalhadores, estas foram distribuídas em três dimensões: indivíduo, equipe e instituição, e perpassam pelo comportamento individual às mudanças estruturais e provisão de equipamentos de trabalho para redução da carga de trabalho.

Assim, com vistas à redução dos DORT, os trabalhadores de enfermagem deveriam coparticiparem na elaboração de medidas institucionais para redução desses agravos, e assim, aumentar as chances de sucesso das estratégias de intervenção para melhoria das condições laborais.

\section{REFERÊNCIAS}

1. Yao Y, Zhao S, An Z, Wang S, Li H, Lu L et al. The associations of work style and physical exercise with the risk of work-related musculoskeletal disorders in nurses. Int. J. Occup. Med. Environ. Health. 2019 [cited 2020 Feb 15]; 32(1):15-24. DOI: https://doi.org/10.13075/ijomeh.1896.01331

2. Brasil. Ministério da Saúde. LER e DORT são as doenças que mais acometem os trabalhadores, aponta estudo. Brasília (DF): Ministério da Saúde; 2019 [cited 2020 Feb 15]. Available from: https://www.saude.gov.br/noticias/agencia-saude/45404-ler-edort-sao-as-doencas-que-mais-acometem-os-trabalhadores-aponta-estudo

3. Galindo IS, Ferreira SCM, Lazzari D, Kempfer SS, Testoni AK. Absenteism reasons in an ambulatorial nursing team. Rev. enferm. UFPE on line. 2017 [cited 2019 Dec 13]; 11:3198-205. Available from: https://periodicos.ufpe.br/revistas/revistaenfermagem/article/view/110184/22064

4. Mota PHS, Lima TA, Berach FR, Schmitt ACB. Impact of musculoskeletal pain in functional disability. Fisioter. Pesqui. 2020 [cited 2020 Apr 12]; 27(1):85-92. DOI: http://dx.doi.org/10.1590/1809-2950/19006327012020.

5. Mininel VA, Felli VEA, Silva EJ, Torri Z, Abreu AP, Branco MTA. Workloads, strain processes and sickness absenteeism in nursing. Rev. latinoam. enferm. (Online). 2013 [cited 2019 Dec 13]; 21(6):1290-7. DOI: http://dx.doi.org/10.1590/0104-1169.2992.2366

6. Kuorinka I, Jonsson B, Kilbom A, Vinterberg H, Biering-Sørensen F, Andersson G et al. Standardised Nordic questionnaires for the analysis of musculoskeletal symptoms. Appl Ergon. 1987 [cited 2019 Dec 13]; 18(3):233-7. DOI: http://dx.doi.org/10.1016/0003-6870(87)90010-x

7. Pinheiro FA, Tróccoli BT, Carvalho CV. Validity of the Nordic Musculoskeletal Questionnaire as morbidity measurement tool Rev. saúde pública (Online). 2002 [cited 2019 Dec 13];36(3):307-12. DOI: http://dx.doi.org/10.1590/S003489102002000300008

8. Barros ENC, Alexandre NMC. Cross-cultural adaptation of the Nordic musculoskeletal questionnaire. Int. Nurs. Rev. 2003 [cited 2019 Dec 13]; 50(2):101-8. DOI: http://dx.doi.org/10.1046/j.1466-7657.2003.00188.x

9. Fernandes CS, Couto G, Carvalho R, Fernandes D, Ferreira P. Self-reported work-related musculoskeletal disorders among health professionals at a hospital in Portugal. Rev. Bras. Med. Trab. 2018 [cited 2019 Dec 13]; 16(3):353-9. DOI: http://dx.doi.org/10.5327/Z1679443520180230

10. Mirmohammadia S, Yazdani J, Etemadinejad S, Asgarinejadd H. A cross-sectional study on work-related musculoskeletal disorders and associated risk factors among hospital health cares. Procedia Manufacturing. 2015 [cited 2019 Dec 13]; 3:4528 34. DOI: https://doi.org/10.1016/j.promfg.2015.07.468

11. Abedini R, Choobineh AR, Hasanzadeh J. Patient manual handling risk assessment among hospital nurses. Work. 2015 [cited 2019 Dec 13]; 50:669-75. DOI: https://doi.org/10.3233/WOR-141826

12. Serranheira F, Sousa-Uva M, Sousa-Uva A. Hospital nurses tasks and work-related musculoskeletal disorders symptoms: a detailed analysis. Work. 2015 [cited 2019 Dec 13];51(3):401-9. DOI: http://dx.doi.org/10.3233/wor-141939

13. Neves AIA, Vieira EMA, Cardia MCG, Lucena NMG, Silva LB. Sociodemographic and organizational factors associated with musculoskeletal symptoms among intensive care unit professionals. Rev. bras. med. trab. 2018 [cited 2019 Dec 13]; 16(3):2639. DOI: http://dx.doi.org/10.5327/z1679443520180240

14. Mauro MYC, Rebelo AMS, Ferreira AOM, Sper NPT, Santos MIS, Gallasch CH. Night work and self-perceived body weight changes among nursing professionals. Rev. enferm. UERJ [Internet]. 2019 [cited 2020 Feb 15]; 27:e31273. DOI: https://doi.org/10.12957/reuerj.2019.31273

15. d'Ettorre G, Vullo A, Pellicani V. Assessing and preventing low back pain in nurses. Implications for practice management. Acta Biomed. 2019 [cited 2020 Feb 15]; 90(6-S):53-9. DOI: https://doi.org/10.23750/abm.v90i6-S.8228

16. Pacheco ES, Sousa ARR, Sousa PTM, Rocha AF. Prevalence of musculoskeletal symptoms related to nursing work in the hospital field. Rev. enferm. UFPI. 2016 [cited 2019 Dec 13]; 5(4):31-7. DOI: https://doi.org/10.26694/reufpi.v5i4.5387

17. Chiwaridzo M, Makotore V, Dambi JM, Munambah N, Mhlanga M. Work-related musculoskeletal disorders among registered general nurses: a case of a large central hospital in Harare, Zimbabwe. BMC Res Notes. 2018 [cited 2019 Dec 13]; 11(1):315. DOI: https://doi.org/10.1186/s13104-018-3412-8

18. Luan HD, Hai NT, Xanh PT, Giang HT, Van Thuc P, Hong NM et al. Musculoskeletal disorders: prevalence and associated factors among district hospital nurses in Haiphong, Vietnam. Biomed Res Int. 2018 [cited 2019 Dec 13]; e3162564. DOI: https://doi.org/10.1155/2018/3162564 
19. Andersen LL, Clausen T, Persson R, Holtermann A. Perceived physical exertion during healthcare work and risk of chronic pain in different body regions: prospective cohort study. Int. Arch. Occup. Environ. Health. 2013 [cited 2019 Dec 13]; 86(6):681-7. DOI: https://doi.org/10.1007/s00420-012-0808-y

20. Silva TPD, Araújo WN, Stival MM, Toledo AM, Burke TN, Carregaro RL. Musculoskeletal discomfort, work ability and fatigue in nursing professionals working in a hospital environment. Rev. Esc. Enferm. USP. 2018 [cited 2019 Dec 13]; 52:e03332. DOI: http://dx.doi.org/10.1590/S1980-220X2017022903332

21. Passali C, Maniopoulou D, Apostolakis I, Varlamis I. Work-related musculoskeletal disorders among Greek hospital nursing professionals: A cross-sectional observational study. Work. 2018 [cited 2019 Dec 13]; 61 (3):489-98. DOI: http://dx.doi.org/10.3233/WOR-182812

22. Járomi M, Kukla A, Szilágyi B, Simon-Ugron Á, Bobály VK, Makai A et al. Back School programme for nurses has reduced low back pain levels: a randomised controlled trial. J. Clin. Nurs. 2018 [cited 2019 Dec 13]; 27:895-902. DOI: http://dx.doi.org/10.1111/jocn.13981

23. Yokota J, Fukutani N, Nin K, Yamanaka H, Yasuda M, Tashiro Y et al. Association of low back pain with presenteeism in hospital nursing staff. J. Occup. Health. 2019 [cited 2020 Jan 22]; 61(3):219-26. DOI: https://doi.org/10.1002/1348-9585.12030

24. Santos HEC, Marziale MHP, Felli VEA. Presenteeism and musculoskeletal symptoms among nursing professionals. Rev. latinoam. enferm. (Online). 2018 [cited 2019 Dec 13]; 26:e3006. DOI: http://dx.doi.org/10.1590/1518-8345.2185.3006

25. Moreira LG, Fernandes M. The presentism in the scope of the nursing in Unit of Intensive Therapy. Revista Pró-univerSUS. 2019 [cited 2020 Jan 22]; 10(1):154-61. DOI: https://doi.org/10.21727/rpu.v10i1.1748

26. Bakola H, Zyga S, Stergioulas U, Kipreos L, Panoutsopoulos L. Musculoskeletal problems among Greek perioperative nurses in regional hospitals in Southern Peloponnese: musculoskeletal problems in perioperative nurses. Adv. Exp. Med. Biol. 2017 [cited 2019 Dec 13]; 989:21-37. DOI: https://doi.org/10.1007/978-3-319-57348-9_3

27. Alencar MCB, Nobre TL. Sickness and suffering of workers due to RSI/WRMSD. Rev. psicol. (Fortaleza, Online). 2017 [cited 2019 Dec 13]; 8(2): 8-18. Available from: http://docs.bvsalud.org/biblioref/2018/01/877146/5861-49919-2-pb.pdf

28. Carvalho DP, Rocha LP, Pinho E, Tomaschewski-Barlem JG, Barlem ELD, Goulart LS. Workloads and burnout of nursing workers. Rev. bras. enferm. 2019 [cited 2020 Feb 18]; 72(6): 1435-41. DOI: http://dx.doi.org/10.1590/0034-7167-2017-0659

29. Dale AP, Dias MDA. The 'extravagance' of working sick: the body work in individuals diagnosed with RSI/WRMD. Trabalho, Educação e Saúde. 2018 [cited 2019 Dec 13]; 16(1): 263-82. DOI: https://dx.doi.org/10.1590/1981-7746-sol00106

30. Lee SJ, Lee JH. Safe patient handling behaviors and lift use among hospital nurses: a cross-sectional study. Int. J. Nurs. Stud. 2017 [cited 2019 Dec 13]; 74:53-60. DOI: https://doi.org/10.1016/j.ijnurstu.2017.06.002

31. Kanaskie ML, Snyder C. Nurses and nursing assistants decision-making regarding use of safe patient handling and mobility technology: a qualitative study. Appl. Nurs. Res. 2018 [cited 2019 Dec 13]; 39:141-7. DOI: https://doi.org/10.1016/j.apnr.2017.11.006

32. Soares MMML, Albino Filho MA, Takeda E, Pinheiro OL. Teacher's perception about the physical ergonomics principles in medicine and nursing courses. Ciênc. cuid. saúde. 2016 [cited 2019 Dec 13]; 15(3):546-52. DOI: http://dx.doi.org/10.4025/cienccuidsaude.v15i3.29384 